\title{
Twist systems on the interval
}

\author{
by \\ Jozef Bobok (Praha) \\ Dedicated to Prof. J. Smital on the occasion of his 60th birthday
}

\begin{abstract}
Let $I$ be a compact real interval and let $f: I \rightarrow I$ be continuous. We describe an interval analogy of the irrational circle rotation that occurs as a subsystem of the dynamical system $(I, f)$-we call it an irrational twist system. Using a coding we show that any irrational twist system is strictly ergodic. We also prove that irrational twist systems exist as subsystems of a large class of systems $(I, f)$ having a cycle of odd period greater than one.
\end{abstract}

0. Introduction. Let $(T, g)$ be a line (resp. interval) dynamical system that consists of a nonempty compact subset (resp. subinterval) $T$ of the real line and a continuous map $g$ mapping $T$ into itself. The question of coexistence of different types of line systems arises in the theory of interval dynamical systems.

For instance, the case when $T$ 's are finite and line systems are created by cycles has been deeply studied [ALM]. In recent works [Bl1-5], [B2-3], [BK3], $[\mathrm{Y}]$ the authors deal with infinite line systems $(\# T=\infty)$ and show interesting results concerning their coexistence.

The aim of this paper is to complete our investigation of twist (line) systems. The class of twist systems can be briefly described as follows: A system $(T, g)$ is said to be ergodic if $T=\operatorname{supp} \mu$ for some ergodic $g$-invariant Borel probability measure. Let $E$ be an eccentricity (function with finite or infinite values, to be introduced later in detail) defined on the set of all line systems. An ergodic system $(T, g)$ is a twist system if there is an interval map $f: \operatorname{conv} T \rightarrow \operatorname{conv} T$ such that $f \mid T=g, E(T, g)=E(\operatorname{conv} T, f)$ and $E(S, f)<E(T, g)$ whenever $S \subset \operatorname{conv} T,(S, f)$ is ergodic and $S \backslash T \neq \emptyset$. In

2000 Mathematics Subject Classification: 26A18, 37A05, 37E05, 37E45.

Key words and phrases: interval map, twist system, invariant measure.

Research supported by the Grant Agency of the Czech Republic, contract no. 201/00/0859. 
other words, it is an ergodic system that does not force more complicated behaviour - as measured by the eccentricity - than is realized by $(T, g)$ itself. Our main result (Theorem 4.5) states that irrational twist systems (i.e., those with irrational eccentricity) are strictly ergodic. In Theorem 5.2 we prove that such systems occur as subsystems of any interval system $(I, f)$ having a cycle of odd period greater than one.

The paper is organized as follows:

In Section 1 we give some basic notation and definitions used throughout the paper.

Section 2 is devoted to the description of useful (known) properties of (twist) systems. An important property of codings of twist cycles is proved in Lemma 2.3 .

Section 3 is devoted to the investigation of rational twist systems. Their study is based on statements 2.1-2.3 and 3.1, 3.3 and Example 3.2. The main result of this section is Theorem 3.4.

In Section 4, using Lemmas 4.1-4.4, we prove that irrational twist systems are strictly ergodic (Theorem 4.5).

Finally, Section 5 is devoted to the proof of Theorem 5.2 that completes the knowledge of twist systems (both rational and irrational) realized by continuous interval maps.

1. Notation, definitions. By $\mathbb{R}, \mathbb{N}, \mathbb{N}_{0}$ we denote the sets of real numbers, positive integers and nonnegative integers respectively. Let $\mathcal{T}$, resp. $\mathcal{I} \subset \mathcal{T}$, be the set of all nonempty compact subsets, resp. subintervals, of $\mathbb{R}$ and let $C(T)$ be the set of all continuous functions which map $T \in \mathcal{T}$ into itself. We consider the space $C(\mathcal{T})=\bigcup\{C(T): T \in \mathcal{T}\}$, resp. $C(\mathcal{I})=\bigcup\{C(I): I \in \mathcal{I}\}$. For $g \in C(\mathcal{T})$ we define $g^{n}$ inductively by $g^{0}=$ id and (for $n \geq 1) g^{n}=g \circ g^{n-1}$.

Let $J$ be a nonempty (maybe one-point) subset of $T \in \mathcal{T}$. The orbit of $J$ under $g$ is $\operatorname{orb}(g, J)=\left\{g^{n}(J)\right\}_{n=0}^{\infty}$. The $\omega$-limit set $\omega(g, x)$ of $x \in T$ consists of all the limit points of $\operatorname{orb}(g, x)$. We will say that a set $J \subset T$ (or a point $x \in T$ ) is $g$-periodic (of period $n$ ) if $J, \ldots, g^{n-1}(J)$ are pairwise disjoint and $g^{n}(J)=J$. A fixed point is a periodic point of period 1 and $\operatorname{Fix}(g)$ is the set of all fixed points of $g$.

For $g \in C(T)$ we will say that $(T, g)$ is a system, resp. interval system for $T \in \mathcal{I}$. For a system $(T, g)$, a map $\widetilde{g} \in C(\operatorname{conv} T)$ is said to be $(T, g)$ monotone if $\widetilde{g} \mid T=g$ and $\widetilde{g} \mid J$ is (not necessarily strictly) monotone for any interval $J \subset \operatorname{conv} T$ such that $J \cap T=\emptyset$. We will use the notation $C(T, g)$ for the set of all $(T, g)$-monotone maps. In particular, the $(T, g)$-monotone map which is affine on each component of $\operatorname{conv} T \backslash T$ will be denoted by $g_{T}$.

An interval map $f \in C(I)$ is piecewise monotone if there are $k \in \mathbb{N}$ and points $\min I=c_{0}<c_{1}<\ldots<c_{k}<c_{k+1}=\max I$ such that $f$ is 
monotone on each $\left[c_{i}, c_{i+1}\right], i=0, \ldots, k$. The minimal such $k$ will be called the modality of $f$, resp. of $(T, g)$ if $f=g_{T}$.

A system $(T, g)$ is said to be minimal, resp. transitive, if $\omega(g, x)=T$ for each $x \in T$, resp. for some $x \in T$. Such a point will be called transitive. A cycle is a minimal system $(T, g)$ for which $T$ is finite. A function $f \in C(\mathcal{I})$ has a system $(T, g)$ if $f \mid T=g$. In this case we will sometimes write $(T, f)$ instead of $(T, g)$.

Let $(T, g)$ be a system. Suppose there are $T$-blocks $B_{i}=\left[a_{i}, b_{i}\right] \cap T$, $i \in\{1, \ldots, k\}$, such that $b_{i}<a_{i+1}$ for $i \in\{1, \ldots, k-1\}$ and $T=\bigcup_{i=1}^{k} B_{i}$. We will say that $(T, g)$ has a block structure over a cycle $(S, f)$ if $S=\left\{s_{1}<\right.$ $\left.\ldots<s_{k}\right\}$, and $g\left(B_{i}\right)=B_{j}$ if and only if $f\left(s_{i}\right)=s_{j}$.

Classification of transitive systems. It is known $[\mathrm{BCp}]$ that for a transitive system $(T, g)$ exactly one of the following three possibilities is satisfied: (i) $T$ is finite; (ii) $T$ is a Cantor set; (iii) $T$ is the union of elements of an orbit of a closed periodic interval.

In what follows we will use some notions from ergodic theory [DGS]. Let $\mu$ be a Borel probability measure on a compact topological space $(X, \tau)$ and let $g: X \rightarrow X$ be continuous. We will say that $\mu$ is $g$-invariant if $\mu\left(g^{-1}(S)\right)=$ $\mu(S)$ for any Borel $S \subset X$. We denote by $\mathcal{M}(g)$ the set of all $g$-invariant measures. A measure $\mu \in \mathcal{M}(g)$ is called ergodic if for any Borel set $S \subset X$ satisfying $g(S) \subset S$ we have either $\mu(S)=0$ or $\mu(S)=1$. We denote the set of all $g$-invariant ergodic measures by $\mathcal{M}_{\mathrm{e}}(g)$. The support of a measure $\mu$, denoted by $\operatorname{supp} \mu$, is the smallest closed set $S \subset X$ such that $\mu(S)=1$. A point $x \in \operatorname{supp} \mu$ is said to be $\mu$-generic if $\lim _{n \rightarrow \infty} n^{-1} \#\{0 \leq i \leq n-1$ : $\left.g^{i}(x) \in U\right\}=\mu(U)$ for each open set $U \subset \operatorname{supp} \mu$ (in the relative topology) such that $\mu(\operatorname{bd} U)=0$. If $G(\mu)$ denotes the set of all $\mu$-generic points then $\mu(G(\mu))=1$, i.e. for an ergodic measure almost every point is generic.

A system $(T, g)$ is said to be ergodic if $T=\operatorname{supp} \mu$ for some $\mu \in \mathcal{M}_{\mathrm{e}}(g)$. An ergodic system is called strictly ergodic if $\# \mathcal{M}_{\mathrm{e}}(g)=1$. It is known that an ergodic, resp. strictly ergodic system is transitive, resp. minimal. We have the following ergodic decomposition.

Theorem $1.1([\mathrm{Ph}])$. Let $g \in C(\mathcal{T})$ and $\mu \in \mathcal{M}(g)$. Then there is a measure $m$ on $\mathcal{M}_{\mathrm{e}}(g)$ such that $\mu(S)=\int_{\mathcal{M}_{\mathrm{e}}(g)} \lambda(S)$ dm for any measurable set $S$.

For our investigation in Section 3 it will be important to know whether for a system $(T, g)$ there are measures in $\mathcal{M}_{\mathrm{e}}(g)$ with support $T$. There is the following remarkable result.

TheOREM 1.2 ([Bl4], [DGS]). Let $(I, f)$ be a transitive interval system. Then $(I, f)$ has the specification property, hence the set of ergodic measures from $\mathcal{M}(f)$ with support $I$ is $G_{\delta}$ dense in $\mathcal{M}(f)$. 
Definition of eccentricity. Let $(T, g)$ be a system, $c \in \operatorname{conv} T$ and $\mu$ be a measure on $T$. Let $\beta=\mu([\min T, c]) / \mu([c, \max T])(\beta=\infty$ if $\mu([c, \max T])=0)$. We define $E(\mu, c)=\max \{\beta, 1 / \beta\}, E(\mu)=\sup \left\{E(\mu, c): c \in \operatorname{Fix}\left(g_{T}\right)\right\}$ and

$$
E(T, g)=\sup \left\{E(\mu): \mu \in \mathcal{M}_{\mathrm{e}}(g)\right\} .
$$

The value $E(T, g)$ will be called the eccentricity of $(T, g)$.

REMARK 1.2. (i) By means of Theorem 1.1 we can verify that $E(T, g)=$ $\sup \{E(\mu): \mu \in \mathcal{M}(g)\}$ for a system $(T, g)$. (ii) If $I \in \mathcal{T}$ is an interval and $f \in C(I)$ then we often write $E(f)$ instead of $E(I, f)$. (iii) As shown in [BK2] if for $g \in C(T)$ the number of fixed points of $g_{T}$ is greater than one then $E\left(g_{T}\right)=\infty$. In this paper our interest is focused on maps with finite eccentricities. So in what follows we will not consider any map $g_{T}$ with more fixed points.

Definition of unisystem. A system $(T, g)$ is said to be a unisystem if $g_{T}$ is piecewise monotone and \# $\operatorname{Fix}\left(g_{T}\right)=1$. The set of all unisystems will be denoted by $\mathcal{U}$.

REMARK 1.3. (i) Let $(T, g) \in \mathcal{U}$. In what follows we always use the letter $c$ to denote the unique fixed point of $g_{T}$. We will also write $T=T_{\mathrm{L}} \cup T_{\mathrm{R}}$ where $T_{\mathrm{L}}=[\min T, c] \cap T$ and $T_{\mathrm{R}}=[c, \max T] \cap T$.

(ii) Our definition of eccentricity gives $E\left(g_{T}\right) \geq E(T, g)$. It is well known that $E\left(g_{T}\right)>E(T, g)$ for some unisystems $(T, g)$ [BK2].

In the following key definition we work with unisystems only.

Definition of twist $(\beta)$-system. Let $\beta \in(1, \infty)$. A system $(T, g) \in \mathcal{U}$ is said to be a twist $(\beta)$-system if $T=\operatorname{supp} \mu$ for some $\mu \in \mathcal{M}_{\mathrm{e}}(g)$ with $E(\mu)=\beta, E\left(g_{T}\right)=\beta$ and

$$
\forall \nu \in \mathcal{M}_{\mathrm{e}}\left(g_{T}\right): \quad E(\nu)=\beta \Rightarrow \operatorname{supp} \nu \subset T .
$$

REMARK 1.4. (i) If $(T, g)$ is a twist $(\beta)$-system then from Theorem 1.1 it follows that

$$
\forall \nu \in \mathcal{M}\left(g_{T}\right): \quad E(\nu)=\beta \Rightarrow \operatorname{supp} \nu \subset T .
$$

(ii) In [BK2] we have studied a particular case of twist systems given by a finite set $T$. In that case $(T, g)$ is a cycle and we will call it the twist cycle. We will show in Section 3 that a twist system $(T, g)$ with a rational eccentricity need not satisfy $\# T<\infty$. Our investigation of twist systems has been extended to the irrational case in [BK3]. The main result of that paper is stated in Proposition 2.2.

REMARK 1.5. It is a consequence of the results proved in [B12] that for $(T, g) \in \mathcal{U}$ with $E\left(g_{T}\right) \in[1, \infty)$ there is some $\mu \in \mathcal{M}_{\mathrm{e}}\left(g_{T}\right)$ such that $E(\mu)=$ 
$E\left(g_{T}\right)$. In what follows we will always assume that

$$
E(\mu)=\mu([\min T, c]) / \mu([c, \max T]) .
$$

Otherwise we would use instead of $g_{T}$ the map $h \circ g_{T} \circ h^{-1}$ with $h(x)=$ $-x+\min T+\max T, x \in[\min T, \max T]$.

Definition of $(\beta)$-code and $(\beta)$-coding. Let $(T, g) \in \mathcal{U}, \beta \in(1, \infty)$. For $x \in T \backslash\{c\}$ a function $K_{x}: \operatorname{orb}(g, x) \rightarrow \mathbb{R}$ is a $(\beta)$-code of $\operatorname{orb}(g, x)$ if for each $i \in \mathbb{N}_{0}$,

$$
K_{x}\left(g^{i+1}(x)\right)= \begin{cases}K_{x}\left(g^{i}(x)\right)+1 /(1+\beta) & \text { if } g^{i}(x)<c \\ K_{x}\left(g^{i}(x)\right)-\beta /(1+\beta) & \text { if } g^{i}(x)>c .\end{cases}
$$

We say that the $(\beta)$-code of $\operatorname{orb}(g, x)$ is monotone, resp. strictly monotone, if for any $y, z \in \operatorname{orb}(g, x)$ the relation $y \in \operatorname{conv}\{z, c\}$ implies $K_{x}(y) \geq K_{x}(z)$, resp. $K_{x}(y)>K_{x}(z)$. A continuous function $K: T \rightarrow \mathbb{R}$ is said to be a $(\beta)$ coding of $(T, g)$ if for each $x \in T \backslash\{c\}$ the function $K \mid \operatorname{orb}(g, x)=K_{x}$ is a $(\beta)$-code of orb $(g, x)$. A $(\beta)$-coding $K$ is monotone, resp. strictly monotone, if so are all codes $K \mid \operatorname{orb}(g, x), x \in T$. A coding $K: T \rightarrow \mathbb{R}$ satisfying $K(\min T)=0$ will be called the normalized coding.

Remark 1.6. By its definition, a coding $K$ of $(T, g)$ (if it exists) is uniquely determined up to an additive constant. In Section 5 we will write $K_{x}(\cdot, \beta)$ to emphasize the choice of $\beta$.

In Section 3 we use symbolic dynamics [DGS]. Consider $\{0,1,2\}$ as a finite space with the discrete topology, and denote by $\Omega_{3}$ the infinite product space $\prod_{i=0}^{\infty} X_{i}$, where $X_{i}=\{0,1,2\}$ for all $i$. The shift map $\sigma: \Omega_{3} \rightarrow \Omega_{3}$ is defined by $(\sigma(\omega))_{i}=\omega_{i+1}, i \in \mathbb{N}_{0}$. Obviously, $\Omega_{3}$ is a compact metrizable topological space and $\sigma: \Omega_{3} \rightarrow \Omega_{3}$ is continuous. Thus, the pair $\left(\Omega_{3}, \sigma\right)$ is a topological dynamics. If a probability vector $p=\left(p_{0}, p_{1}, p_{2}\right)$ with positive coordinates defines a measure $\nu$ on $\{0,1,2\}$ satisfying $\nu\{i\}=p_{i}$ then the corresponding product measure $\lambda=\prod_{i=0}^{\infty} \nu$ on $\Omega_{3}$ (Bernoulli measure given by $p$ ) is $\sigma$-invariant, ergodic and $\operatorname{supp} \lambda=\Omega_{3}$.

2. Useful properties of (twist) systems. This section is devoted to the description of the necessary auxiliary results. Important known properties of twist cycles are listed in the next proposition. Statement (iv) is a special case of a more general one proved in [Bl2].

Proposition 2.1. (i) [BK2] A cycle $(T, g)$ is a twist $(\beta)$-cycle if and only if there is a strictly monotone $(\beta)$-coding of $(T, g)$. In this case if $\beta=$ $m / n$ with $m, n$ coprime then $(T, g)$ has a period $m+n$.

(ii) [Bl5] Let $f \in C(\mathcal{I})$ and $\beta \in[1, E(f))$ be rational. Then $f$ has a twist ( $\beta)$-cycle. 
(iii) [B1, 3.2(ii)] Let $\left(T=\left\{t_{1}<\ldots<t_{k}\right\}, g\right)$ be a twist cycle. If $(T, g)$ has a block structure over a cycle $\left(S=\left\{s_{1}<\ldots<s_{l}\right\}\right.$, f) with $l>1$ then $k=l$, and $g\left(t_{i}\right)=t_{j}$ if and only if $f\left(s_{i}\right)=s_{j}$. Moreover, $\left(\operatorname{conv} T, g_{T}\right)$ is transitive.

(iv) [Bl2] Let $(T, g)$ be piecewise monotone. If the eccentricity $E\left(g_{T}\right)>1$ of $g_{T}$ is rational then $g_{T}$ has a twist $E\left(g_{T}\right)$-cycle.

(v) [BK3] If $(T, g)$ is a twist $(\beta)$-system for $\beta \in(1, \infty)$ then $T \cap$ $\operatorname{Fix}\left(g_{T}\right)=\emptyset$.

The next proposition recalls the main results describing twist systems with irrational eccentricity. Of course, in this case the set $T$ carrying an irrational system $(T, g)$ has to be infinite.

Proposition 2.2 ([BK3]). Let $(T, g) \in \mathcal{U}$ and let $\beta \in(1, \infty)$ be irrational. The following statements are equivalent.

(i) $(T, g)$ is a twist $(\beta)$-system.

(ii) $T=\operatorname{supp} \mu$ for some $\mu \in \mathcal{M}_{\mathrm{e}}(g)$ with $E(\mu)=\beta$ and there is a map $\widetilde{g} \in C(T, g)$ such that $E(\widetilde{g})=\beta$.

(iii) There is a transitive point $x \in T$ such that the $(\beta)$-code $K_{x}$ of $\operatorname{orb}(g, x)$ is monotone.

(iv) $(T, g)$ is minimal and there is a $(\beta)$-coding $K: T \rightarrow \mathbb{R}$ such that for each $x \in T$, the $(\beta)$-code $K_{x}=K \mid \operatorname{orb}(g, x)$ of $\operatorname{orb}(g, x)$ is strictly monotone.

(v) $T=\operatorname{supp} \mu$ for some $\mu \in \mathcal{M}_{\mathrm{e}}(g)$ with $E(\mu)=\beta$, for any $\widetilde{g} \in C(T, g)$ we have $E(\widetilde{g})=\beta$ and

$$
\forall \nu \in \mathcal{M}(\widetilde{g}): \quad E(\nu)=\beta \Rightarrow \operatorname{supp} \nu=T .
$$

Let $(T, g)$ be a twist $(\beta)$-cycle. Using Proposition 2.1(i) we can verify that the modality $M(T, g)$ of $(T, g)$ is odd; Remark 1.5 implies that $g\left(T_{\mathrm{R}}\right) \subset T_{\mathrm{L}}$ (both properties easily follow from the fact that the $(\beta)$-coding of $(T, g)$ is strictly monotone). Put $M(T, g)=2 j-1 \in \mathbb{N}$, write $T_{\mathrm{R}}=T_{0}$ and express $T_{\mathrm{L}}$ as a union of $2 j$ consecutive blocks, i.e.

$$
T_{\mathrm{L}}=\bigcup_{1 \leq i \leq j} T_{2 i-1} \cup T_{2 i}
$$

where for $1 \leq i \leq j$ each block with odd (resp. even) index is mapped by $g$ into $T_{\mathrm{L}}$ (resp. $T_{\mathrm{R}}$ ) and they are ordered (from the left) according to their labels (see also [B1, Section 3]).

The following statement will be important when proving Theorem 5.2. It provides some extra information on codings of twist cycles and we present it in this summarizing section.

Lemma 2.3. Let $(T, g)$ be a twist $(\beta)$-cycle with modality $M(T, g)=$ $2 j-1$, and denote by $K$ its normalized $(\beta)$-coding. Then $\max K \mid T_{2 k}<$ $k \beta /(1+\beta)$ for each $k \in\{1, \ldots, j\}$. In particular, $\max K \mid T_{\mathrm{L}}<j \beta /(1+\beta)$. 
Proof. Since by Proposition 2.1(i) the $(\beta)$-coding $K: T \rightarrow \mathbb{R}$ is strictly monotone we must have

$$
g \mid \bigcup_{1 \leq i \leq j} T_{2 i-1} \text { is increasing \& } g \mid \bigcup_{0 \leq i \leq j} T_{2 i} \text { is decreasing. }
$$

Using (1) we have for each $x \in \bigcup_{1 \leq i \leq j} T_{2 i}$ the relations

$$
K\left(g^{2}(x)\right)=K(x)+\frac{1-\beta}{1+\beta}<K(x),
$$

hence $g^{2}(x)<x$.

Set $x_{i}=\min T_{i}$ and $y_{i}=\max T_{i}$ for $i \in\{1, \ldots, 2 j\}$. We are going to show that for each $i \in\{1, \ldots, j\}$ :

(i) $g\left(y_{2 i-1}\right) \geq y_{2 i}$ with equality iff $i=j$,

(ii) $K\left(y_{2 j}\right)-K\left(y_{2 j-1}\right)=1 /(1+\beta)$ and $K\left(y_{2 i}\right)-K\left(y_{2 i-1}\right) \leq 1 /(1+\beta)$ for $i<j$,

(iii) $g^{2}\left(x_{2 i}\right) \leq x_{2 i-1}$ with equality iff $i=1$,

(iv) $K\left(x_{2}\right)-K\left(x_{1}\right)=(\beta-1) /(1+\beta)$ and $K\left(x_{2 i}\right)-K\left(y_{2 i-2}\right) \leq(\beta-1) /$ $(1+\beta)$ for $i>1$.

Let us prove (i). Clearly for each $x \in \bigcup_{1<i<j} T_{2 i}$ we have $g^{2}(x)<$ $g^{2}\left(y_{2 j}\right)<y_{2 j}$. Since $g(T)=T$, from (2) we obtain $g\left(y_{2 j-1}\right)=y_{2 j}$.

Let $i<j$ and suppose that $g\left(y_{2 i-1}\right) \leq y_{2 i}$. Then putting $S=\left(T_{1} \cup\right.$ $\left.T_{2}\right) \cup \ldots \cup\left(T_{2 i-1} \cup T_{2 i}\right)$ we get from (2) the relations $S \cup g(S) \subsetneq T$ and $g(S \cup g(S)) \subset S \cup g(S)$, which contradicts $g(T)=T$. Thus $g\left(y_{2 i-1}\right)>y_{2 i}$.

Property (ii) follows directly from (i).

In order to prove (iii) we start by showing that $g^{2}\left(x_{2}\right)=x_{1}$. Indeed, by (2) we have $g\left(x_{2}\right)=\max T$ and $g^{2}\left(x_{2}\right)=g(\max T)=\min T=x_{1}$.

Let $i>1$ and suppose that $g^{2}\left(x_{2 i}\right) \geq x_{2 i-1}$. Define $R=\left(T_{2 i-1} \cup T_{2 i}\right) \cup$ $\ldots \cup\left(T_{2 j-1} \cup T_{2 j}\right)$. Then by (2) we obtain $R \cup g(R) \subsetneq T$ and $g(R \cup g(R)) \subset$ $R \cup g(R)$ - a contradiction. This means $g^{2}\left(x_{2 i}\right)<x_{2 i-1}$.

Property (iv) follows easily from (iii).

Fix $k \in\{1, \ldots, j\}$. Using $K\left(x_{1}\right)=0$ we have

$$
\begin{aligned}
K\left(y_{2 k}\right)= & \sum_{i=1}^{k-1}\left(K\left(y_{2 i+2}\right)-K\left(x_{2 i+2}\right)+K\left(x_{2 i+2}\right)-K\left(y_{2 i}\right)\right) \\
& +K\left(y_{2}\right)-K\left(x_{2}\right)+K\left(x_{2}\right)-K\left(x_{1}\right) \\
= & \sum_{i=0}^{k-1}\left(K\left(y_{2 i+2}\right)-K\left(x_{2 i+2}\right)\right) \\
& +K\left(x_{2}\right)-K\left(x_{1}\right)+\sum_{i=1}^{k-1}\left(K\left(x_{2 i+2}\right)-K\left(y_{2 i}\right)\right) ;
\end{aligned}
$$


by (ii) we can verify that $K\left(y_{2 i+2}\right)-K\left(x_{2 i+2}\right)<1 /(1+\beta)$ for each $i \in$ $\{0, \ldots, k-1\}$, and similarly it follows from (iv) that $K\left(x_{2}\right)-K\left(x_{1}\right)=$ $(\beta-1) /(1+\beta)$ and for each $i \in\{1, \ldots, k-1\}$ we have $K\left(x_{2 i+2}\right)-K\left(y_{2 i}\right) \leq$ $(\beta-1) /(1+\beta)$. Summarizing, (3) together with the last inequalities gives

$$
K\left(y_{2 k}\right)<k \frac{1}{1+\beta}+k \frac{\beta-1}{1+\beta}=k \frac{\beta}{1+\beta} .
$$

Since $\max K \mid T_{2 k}=K\left(y_{2 k}\right)$ the conclusion follows.

Corollary. Let $(T, g)$ be a twist $(\beta)$-cycle with modality $M(T, g)=$ $2 j-1$, and denote by $K$ its $(\beta)$-coding. Then for each $x, y \in T$ we have

$$
K(x)-K(y)<\frac{j \beta+1}{1+\beta} .
$$

Proof. By (1) and Proposition 2.1(i) we have

$$
\max K=K\left(\min T_{\mathrm{R}}\right)=K\left(\max T_{\mathrm{L}}\right)+\frac{1}{1+\beta}
$$

and $\max T_{\mathrm{L}}=y_{2 j}$. Without loss of generality we can assume that $K$ is normalized. Then by Lemma 2.3 for each $x, y \in T$ we obtain

$$
K(x)-K(y) \leq \max K<j \frac{\beta}{1+\beta}+\frac{1}{1+\beta} .
$$

REMARK. Thus we have shown that the modality of a twist cycle is closely connected with a bound for its coding. For more detailed results of this type see $[\mathrm{BM}]$.

Lemma 2.4. Let $(T, g)$ be a system.

(i) For every interval $J \subset \operatorname{conv} T$ we have

$$
\operatorname{conv}(J \cap T)=J \Rightarrow \operatorname{conv}\left(g_{T}(J) \cap T\right)=g_{T}(J) .
$$

(ii) $[\mathrm{Pr}]$ If $(T, g)$ is transitive, $g_{T}$ piecewise monotone and for some $R \subset T$ the system $(R, g)$ is a cycle then there is a transitive $g_{T}$-periodic interval $[a, b]$ such that $T \subset \bigcup \operatorname{orb}\left(g_{T},[a, b]\right)$.

Proof. (i) This follows directly from the definitions of $(T, g)$ and $g_{T}$.

3. Rational twist systems. In [BK3] we have described some basic properties of irrational twist systems. Theorem 3.4 and further results of this section provide an analogous description for rational eccentricities. In particular, we find that our definition of a twist system $(T, g)$ does not imply $\# T<\infty$ in the rational case.

Let $g \in C(T)$. If the system ( $\left.\operatorname{conv} T, g_{T}\right)$ is transitive we will briefly say that $g_{T}$ is transitive. It is known [BCp] that a piecewise monotone map $g_{T}$ with $E\left(g_{T}\right)>1$ is transitive if and only if (for $c<d$ )

$$
\forall[c, d] \subset \operatorname{conv} T \exists m \in \mathbb{N}: \quad g_{T}^{m}([c, d])=\operatorname{conv} T .
$$


We will also say that $J \subset \operatorname{conv} T$ is a transitive $g_{T}$-periodic interval whenever it is a $g_{T}$-periodic interval of period $n$ for which the system $\left(J, g_{T}^{n}\right)$ is transitive. Then the set $\operatorname{Per}\left(g_{T}\right) \cap J$ is dense in $J$. Note that such an interval is maximal, i.e. there is no other transitive $g_{T}$-periodic interval $I \subset \operatorname{conv} T$ such that $I \supsetneq J$.

LEMmA 3.1. Let $(T, g)$ be a twist $(\beta)$-system for $\beta \in(1, \infty)$ rational. If $g_{T}$ is not transitive then $T$ is the union of elements of the orbit of a transitive $g_{T}$-periodic interval and the system $(T, g)$ has a block structure over a twist $(\beta)$-cycle.

Proof. Let $\beta=m / n, m, n$ coprime. It follows from Proposition 2.1(iv) that $g_{T}$ has some twist $(\beta)$-cycle $(R, g)$; this notation is correct since by $(\star)$ in the definition of twist system and Proposition 2.1(iii) we have $R \subsetneq T$. This means that $\# T=\infty$ and $(T, g)$ is transitive (as the support of an ergodic measure) but not minimal.

By Lemma 2.4(ii) there is a transitive $g_{T}$-periodic interval $[a, b]$ of period $k>1$ such that $T \subset \bigcup \operatorname{orb}\left(g_{T},[a, b]\right)$. Using Proposition 2.1(iii) we see that $\#\left(R \cap g_{T}^{i}([a, b])\right)=1$ for each $i \in\{0, \ldots, k-1\}$, and (i) of the same proposition shows that $k=m+n$. Obviously all measures supported by periodic orbits in $\bigcup \operatorname{orb}\left(g_{T},[a, b]\right)$ have eccentricity $\beta$, hence by the definition of a twist system, $T=\bigcup \operatorname{orb}\left(g_{T},[a, b]\right)$. Moreover, $(T, g)$ has a block structure over $(R, g)$.

In the previous lemma we assumed that for a rational twist system $(T, g)$ the map $g_{T}$ was not transitive. By Proposition 2.1(iii) this is not true for $T$ finite. In what follows we show that $g_{T}$ can be transitive even for infinite $T$. Our example of this phenomenon requires a more detailed explanation. The reason is that such a rational twist system $(T, g)$ has to be ergodic (not minimal) and satisfy condition $(\star)$ from Section 1 .

Example 3.2. Consider the cycle $(S, f)$ given by $S=\{1,2,3,4,5,6\}$ and $f(1)=3, f(2)=6, f(3)=4, f(4)=5, f(5)=2, f(6)=1$. Then $E(S, f)=2$ and $(S, f) \in \mathcal{U}$. Moreover, $(S, f)$ has a monotone (but not strictly monotone) (2)-coding and $E\left(f_{S}\right) \geq 2$ (we will see in part I of the next proof that $\left.E\left(f_{S}\right)=2\right)$ for $f_{S} \in C([1,6])$. It follows from Proposition 2.1(i) that the cycle $(S, f)$ is not a twist (2)-cycle, hence again from Proposition 2.1(i),(ii),(iv), $f_{S}$ has a cycle of period 3 (that is, a twist (2)-cycle). It is known $[\mathrm{MN}]$ that the map $f_{S}$ is transitive. Put

$$
\mathcal{M}=\left\{\nu \in \mathcal{M}_{\mathrm{e}}\left(f_{S}\right): E(\nu)=2\right\}, \quad T=\overline{\bigcup_{\nu \in \mathcal{M}} \operatorname{supp} \nu}, \quad g=f_{S} \mid T .
$$

Lemma 3.3. If $(T, g)$ is as above then $T$ is a Cantor set and $(T, g)$ is a twist (2)-system. 
Proof. Clearly $(T, g)$ is a system and $g_{T}=f_{S}$, hence $(T, g) \in \mathcal{U}$. By the definition of $T$ if $\nu \in \mathcal{M}_{\mathrm{e}}\left(g_{T}\right)$ and $E(\nu)=2$ then $\operatorname{supp} \nu \subset T$. Thus, it is sufficient to show that $T$ is a Cantor set and $T=\operatorname{supp} \mu$ for some $\mu \in \mathcal{M}_{\mathrm{e}}(g)$ with $E(\mu)=2$. The proof will be divided into several parts.

Since $g_{T}=f_{S}$, the map $g_{T}$ is transitive. Denote by $C, D$ the subsets of $[1,6]$ defined by $C=\left\{y: g_{T}^{j}(y) \neq c\right.$ for $\left.j \in \mathbb{N}_{0}\right\}, D=\left\{y: g_{T}^{j}(y) \notin S\right.$ for $j \in$ $\left.\mathbb{N}_{0}\right\}$, and set $I_{0}=[c, 5], I_{1}=[5,6], I_{2}=[1,2], I_{3}=[2,3], I_{4}=[3,4]$, $I_{5}=[4,5]$. A subset $J \subset[1,6]$ covers a subset $L \subset[1,6]$ if $L \subset g_{T}(J)$. In this case we write $J \rightarrow L$. The reader can verify that the covering properties of $I_{0}, I_{1}, I_{2}, I_{3}, I_{4}, I_{5}$ are $I_{0} \rightarrow I_{3} \cup I_{4} \cup I_{5}, I_{1} \rightarrow I_{2}, I_{2} \rightarrow I_{0} \cup I_{1} \cup I_{4} \cup I_{5}$, $I_{3} \rightarrow I_{0} \cup I_{1} \cup I_{5}, I_{4} \rightarrow I_{0} \cup I_{5}, I_{5} \rightarrow I_{0}$.

I. The symbolic itinerary $I(y)=y(0) y(1) \ldots y(j) \ldots$ of $y \in C$ satisfies

$$
y(j) \in\{0,1\} \&\left(c-g_{T}^{j}(y)\right)(-1)^{y(j)}>0 \quad \text { for each } j \in \mathbb{N}_{0} .
$$

For $k \in \mathbb{N}$ and $r(k), s(k), t(k) \in \mathbb{N}_{0}$ put

$$
A(k)=(010001)_{r(k)}(001)_{s(k)}(01)_{t(k)},
$$

where ()$_{m}$ denotes the $m$-fold repetition of the bracketed term. Then using the covering properties of $I_{0}, I_{1}, I_{2}, I_{3}, I_{4}, I_{5}$ we find that for any $y \in C \cap[1, c)$ there are sequences $\{r(y, k)\},\{s(y, k)\},\{t(y, k)\}$ depending on $y$ such that $I(y)$ can be written as

$$
I(y)=A(1) A(2) \ldots A(k) \ldots
$$

Notice that as a consequence we obtain $E\left(g_{T}\right)=E\left(f_{S}\right)=2$.

II. Let $\nu \in \mathcal{M}_{\mathrm{e}}\left(g_{T}\right)$ be such that $E(\nu)=2$ and fix $x \in G(\nu) \cap[1, c)$. Since $x$ is generic, its itinerary $I(x)=x(0) x(1) \ldots x(j) \ldots$ satisfies

$$
E(\nu)=2=\lim _{n \rightarrow \infty} \frac{\sum_{j=0}^{n-1} 1-x(j)}{\sum_{j=0}^{n-1} x(j)}=\lim _{n \rightarrow \infty} \frac{\#\{0 \leq j \leq n-1: x(j)=0\}}{\#\{0 \leq j \leq n-1: x(j)=1\}} ;
$$

by the above we can write $I(x)=A(1) A(2) \ldots A(k) \ldots$ Since $x$ is generic, by (4) it can be easily seen that $t(x, k)=0$, i.e. $A(k)=(010001)_{r(x, k)}(001)_{s(x, k)}$ for each $k \in \mathbb{N}$.

III. Thus, the symbolic itinerary $I(x)$ of $x \in G(\nu) \cap[1, c)$ is built up from two blocks (010001) and (001). These blocks correspond to the blocks of covering intervals. Namely, using the symbols $J \rightarrow L$ if $L \subset g_{T}(J)$, and $(J K \ldots M)$ if $J \rightarrow K \rightarrow \ldots \rightarrow M$, we have the following relations between finite symbolic and interval blocks (draw a picture):

$$
(010001) \leftrightarrow\left(I_{3} I_{1} I_{2} I_{4} I_{5} I_{0}\right), \quad(001) \leftrightarrow\left\{\begin{array}{l}
\left(I_{3} I_{5} I_{0}\right), \\
\left(I_{4} I_{5} I_{0}\right), \\
\left(I_{2} I_{4} I_{0}\right), \\
\left(I_{2} I_{5} I_{0}\right) .
\end{array}\right.
$$


Assume that $x \in D$. As in part I, the interval itinerary $J(x)=J(0) J(1) \ldots$ $\ldots J(j) \ldots$ of $x$ satisfies

$$
J(j) \in\left\{I_{0}, I_{1}, I_{2}, I_{3}, I_{4}, I_{5}\right\} \& g_{T}^{j}(x) \in \operatorname{int} J(j) \quad \text { for each } j \in \mathbb{N}_{0} .
$$

Let us show that $J(x)$ cannot contain blocks $\left(I_{2} I_{4} I_{0}\right)$ and $\left(I_{2} I_{5} I_{0}\right)$. To the contrary, suppose $J(x)$ contains $\left(I_{2} I I_{0}\right), I \in\left\{I_{4}, I_{5}\right\}$, as a block $(J(j) J(j+1) J(j+2))$. Then we can suppose that $j \geq 3$ ( $x$ is generic) and $J(x)$ has to contain the interval block $\left(K J I_{1} I_{2} I I_{0}\right)$, where $(K J) \in$ $\left\{\left(I_{1} I_{2}\right),\left(I_{0} I_{3}\right)\right\}$. In any case the corresponding symbolic block of $I(x)$ is (101001), which contradicts the result of part II.

IV. Consider the symbolic dynamics $\left(\Omega_{3}, \sigma\right)$ and let $\lambda \in \mathcal{M}_{\mathrm{e}}(\sigma)$ satisfy $\operatorname{supp} \lambda=\Omega_{3}$ (see Section 1). Let $\omega=(\omega(0), \omega(1), \ldots)$ be a generic point for the measure $\lambda$. There is a sequence $\{j(k)\}_{k \in \mathbb{N}}$ satisfying

(i) $0=j(1)<j(2)<\ldots$,

(ii) $\omega(j(k)) \leq \omega(j(k)+1) \leq \ldots \leq \omega(j(k+1)-1)$ and $\omega(j(k+1)-1)>$ $\omega(j(k+1))$ for each $k \in \mathbb{N}$.

For each $k \in \mathbb{N}$ put

$$
\begin{aligned}
r(k) & =\#\{j(k) \leq i \leq j(k+1)-1: \omega(i)=0\}, \\
s 1(k) & =\#\{j(k) \leq i \leq j(k+1)-1: \omega(i)=1\}, \\
s 2(k) & =\#\{j(k) \leq i \leq j(k+1)-1: \omega(i)=2\} .
\end{aligned}
$$

We know that $I_{0} \rightarrow I_{3}$ and $I_{0} \rightarrow I_{4}$. This means that we can consider the interval itinerary $J=B(1) B(2) \ldots B(k) \ldots$, where

$$
B(k)=\left(I_{3} I_{1} I_{2} I_{4} I_{5} I_{0}\right)_{r(k)}\left(I_{3} I_{5} I_{0}\right)_{s 1(k)}\left(I_{4} I_{5} I_{0}\right)_{s 2(k)} .
$$

Since the map $g_{T}$ is transitive there exists a unique $x \in[1,6]$ such that $J(x)=J$. We assume that $\omega \in \Omega_{3}$ is generic for the measure $\lambda \in \mathcal{M}_{\mathrm{e}}(\sigma)$ and $g_{T}$ is transitive. The following facts are rather easy consequences of parts I-III. We leave their verification to the reader.

(i) $x \in \omega(g, x)$. (Hint: $\omega$ is a transitive point in $\left(\Omega_{3}, \sigma\right)$.)

(ii) $\omega(g, x)=T$ and $T$ is a Cantor set. (Hint: Parts II-III.)

(iii) There is a measure $\mu \in \mathcal{M}_{\mathrm{e}}(g)$ such that $T=\operatorname{supp} \mu=\omega(g, x)$. (Hint: $\lambda \in \mathcal{M}_{\mathrm{e}}(\sigma)$ and $\operatorname{supp} \lambda=\Omega_{3}$.)

This proves the lemma.

REMARK. The reader should notice that the measure $\mu$ from (iii) depends on the choice of $\lambda$.

We now state the main result of this section. In its formulation we use the decomposition $T=T_{\mathrm{L}} \cup T_{\mathrm{R}}$ introduced in Remark 1.3.

Theorem 3.4. Let $(T, g) \in \mathcal{U}$ and $\beta \in(1, \infty)$ be rational. The following statements are equivalent. 
(i) $(T, g)$ is a twist $(\beta)$-system and $T$ is not a Cantor set.

(ii) The set $T$ is the union of elements of the orbit of a transitive $g_{T^{-}}$ periodic (maybe one-point) interval and the system $(T, g)$ has a block structure over a twist $(\beta)$-cycle. All points of this cycle can be taken as the maxima, resp. minima, of the connected components of $T_{\mathrm{L}}$, resp. $T_{\mathrm{R}}$. In particular, there is a $(\beta)$-coding $K: T \rightarrow \mathbb{R}$ such that for each $x \in T$, the $(\beta)$-code $K_{x}=K \mid \operatorname{orb}(g, x)$ of $\operatorname{orb}(g, x)$ is monotone.

Proof. By Proposition 2.1 the conclusion is true for any system $(T, g) \in$ $\mathcal{U}$ with $T$ finite. So suppose that $\# T=\infty$ and $\beta=m / n$ for $m, n$ coprime.

(i) $\Rightarrow$ (ii). By the classification of transitive systems presented in Section 1 and Proposition 2.1(v) the map $g_{T}$ is not transitive. Let $\left[a_{1}=\min T, b_{1}\right]$ be a transitive $g_{T}$-periodic interval of period $k=m+n>1$ ensured by Lemma 3.1. Denote by $J_{1}=\left[a_{1}, b_{1}\right]<\ldots<J_{k}=\left[a_{k}, b_{k}\right]$ the spatially ordered connected components of $T=\bigcup \operatorname{orb}\left(g_{T}, J_{1}\right)$ and consider $(R=$ $\left.\left\{r_{1}<\ldots<r_{k}\right\}, g\right)$ as in the proof of Lemma 3.1. From Lemma 3.1 we know that $J_{i}=J_{i} \cap T, i \in\{1, \ldots, k\}$, and

$$
g_{T}\left(J_{i}\right)=J_{j} \Leftrightarrow g\left(J_{i}\right)=J_{j} \Leftrightarrow g\left(r_{i}\right)=r_{j} .
$$

Using (1) and Proposition 2.1(i) we can consider a strictly monotone $\left(\frac{n}{m+n}\right)$ coding $K_{R}$ of $(R, g)$ such that $K_{R}\left(r_{1}\right)=0$. We have seen that $R \subset T$ and the map $K_{R}$ can be extended to an $\left(\frac{n}{m+n}\right)$-coding $K: T=\bigcup \operatorname{orb}\left(g_{T}, J_{1}\right) \rightarrow \mathbb{R}$ by $K\left(J_{i}\right)=K_{R}\left(r_{i}\right), i=1, \ldots, k$. Clearly $K$ is still monotone, but not strictly since it is constant on each interval $J_{i}$. Let $M_{i}, i=1, \ldots, m+n$, be closed $(T \cup\{c\})$-contiguous intervals labeled from the left, i.e. $M_{i}=\left[b_{i}, a_{i+1}\right]$ for $i \in\{1, \ldots, m-1\}, M_{i+1}=\left[b_{i}, a_{i+1}\right]$ for $i \in\{m+1, \ldots, m+n-1\}$ and $M_{m}=\left[b_{m}, c\right], M_{m+1}=\left[c, a_{m+1}\right]$. Clearly

$$
M_{1}<\ldots<M_{m-1}<M_{m} \leq c \leq M_{m+1}<\ldots<M_{m+n} .
$$

Since $\min M_{i} \in T$ for $i \leq m$ and $\max M_{i} \in T$ when $i>m$ we can define the $\operatorname{map} \mathrm{E}:\left\{M_{i}\right\}_{i=1}^{m+n} \rightarrow \mathbb{R}$ by

$$
\mathrm{E}\left(M_{i}\right)= \begin{cases}K\left(\min M_{i}\right), & 1 \leq i \leq m, \\ K\left(\max M_{i}\right), & m<i \leq m+n .\end{cases}
$$

By the definitions of $K$ and $\mathrm{E}$ we have for each $r \in R$ the implication

$$
\left(\mathrm{E}\left(M_{i}\right)=K(r) \& \mathrm{\textrm {L }}\left(M_{j}\right)=K(g(r))\right) \Rightarrow g_{T}\left(M_{i}\right) \supset M_{j} .
$$

Using that we can see the following: There exists a closed interval $J \subset M_{1}$ for which $g_{T}^{j}(J) \subset M \in\left\{M_{i}\right\}_{i=1}^{m+n}$ with $\mathrm{\textrm {L }}(M)=K\left(g^{j}\left(r_{1}\right)\right)$ for each $0 \leq j<$ $m+n$ and $J \subset g_{T}^{m+n}(J)=M_{1}$. In other words, $\bigcup \operatorname{orb}\left(g_{T}, J\right) \subset \bigcup_{i=1}^{m+n} M_{i}$ and $\bigcup \operatorname{orb}\left(g_{T}, J\right)$ contains some cycle $\left(S, g_{T}\right)$ with a strictly monotone $\left(\frac{n}{m+n}\right)$ coding. By Proposition 2.1(i), $\left(S, g_{T}\right)$ is a twist $(\beta)$-cycle corresponding to the same cyclic permutation as $(R, g)$. Since the system $(T, g)$ has a block structure over $(R, g)$, the interval $J \subset M_{1}$ can be chosen in such a way 
that $\max J<a_{2}$. Supposing that the choice of points of $S$ described in our statement is not possible we find that also $\min J>b_{1}$. But then $S \cap T=\emptyset$ a contradiction. This proves the first part of our theorem.

(ii) $\Rightarrow$ (i). By our assumption $(T, g) \in \mathcal{U}$. Suppose that (ii) is satisfied. Then Theorem 1.2 shows that for some $\mu \in \mathcal{M}_{\mathrm{e}}(g)$ we have $T=\operatorname{supp} \mu$ and $E(\mu)=\beta$. Thus, we need to verify that $E\left(g_{T}\right)=\beta$ and the property $(\star)$.

Denote by $\left(R=\left\{r_{1}<\ldots<r_{m+n}\right\}, g\right)$ the guaranteed cycle created by maxima and minima of $M$ 's. Choose some $\nu \in \mathcal{M}_{\mathrm{e}}\left(g_{T}\right)$ with $\operatorname{supp} \nu \backslash T \neq \emptyset$. Then for a $\nu$-generic point $y \in \operatorname{supp} \nu \cap M_{j}$ we have $\operatorname{orb}\left(g_{T}, y\right) \cap T=\emptyset$ and there is a unique sequence $\left\{N_{i}\right\}_{i \in \mathbb{N}_{0}}$ of elements of $\left\{M_{i}\right\}_{i=1}^{m+n}$ (with $N_{0}=M_{j}$ ) such that $y_{i}=g_{T}^{i}(y) \in \operatorname{int} N_{i}$ for each $i \in \mathbb{N}_{0}$.

Notice the following property implied by monotonicity of the $(\beta)$-coding $K$ : if $g_{T}\left(N_{i}\right) \supset N_{j}$ then

$$
\mathrm{E}\left(N_{j}\right) \geq \begin{cases}\mathrm{L}\left(N_{i}\right)+\frac{1}{1+\beta} & \text { for } N_{i}<c, \\ \mathrm{E}\left(N_{i}\right)-\frac{\beta}{1+\beta} & \text { for } N_{i}>c .\end{cases}
$$

Clearly $g_{T}\left(N_{i}\right) \supset N_{i+1}$. By the above, if we put

$$
\mathrm{L}\left(N_{i+1}\right)= \begin{cases}\mathrm{L}\left(N_{i}\right)+\frac{1}{1+\beta}+\varepsilon\left(y_{i}\right) & \text { for } N_{i}<c, \\ \mathrm{~L}\left(N_{i}\right)-\frac{\beta}{1+\beta}+\varepsilon\left(y_{i}\right) & \text { for } N_{i}>c,\end{cases}
$$

then $\left\{\varepsilon_{i}=\varepsilon\left(y_{i}\right)\right\}_{i \geq 0}$ is a sequence with finitely many nonnegative values, and for each $n \in \mathbb{N}$ we can write

$$
\begin{aligned}
\frac{1}{n} \mathrm{E}\left(N_{n}\right)= & \frac{1}{n} \mathrm{E}\left(N_{0}\right)+\frac{1}{1+\beta} \frac{1}{n} \sum_{i=0}^{n-1} \chi_{\mathrm{L}}\left(y_{i}\right) \\
& -\frac{\beta}{1+\beta} \frac{1}{n} \sum_{i=0}^{n-1} \chi_{\mathrm{R}}\left(y_{i}\right)+\frac{1}{n} \sum_{i=0}^{n-1} \varepsilon_{i} .
\end{aligned}
$$

Here $\chi_{\mathrm{L}}$, resp. $\chi_{\mathrm{R}}$ is the indicator function of $[\min T, c], \operatorname{resp} .[c, \max T]$.

Clearly $\lim _{n} n^{-1} \mathrm{~L}\left(N_{n}\right)=\lim _{n} n^{-1} \mathrm{\textrm {L }}\left(N_{0}\right)=0$. Now we use the fact that $y$ is $\nu$-generic. Obviously the monotonicity of $K$ gives $E(\nu)=\gamma=$ $\nu([\min T, c]) / \nu([c, \max T])$ and then

$$
\lim _{n \rightarrow \infty} \frac{1}{n} \sum_{i=0}^{n-1} \chi_{\mathrm{L}}\left(y_{i}\right)=\frac{\gamma}{1+\gamma} \& \lim _{n \rightarrow \infty} \frac{1}{n} \sum_{i=0}^{n-1} \chi_{\mathrm{R}}\left(y_{i}\right)=\frac{1}{1+\gamma} .
$$

Putting the last limits into (5) we get

$$
0 \leq \lim _{n} \frac{1}{n} \sum_{i=0}^{n-1} \varepsilon_{i}=\frac{\beta-\gamma}{(1+\beta)(1+\gamma)} .
$$

Now we can see that $\gamma<\beta$ if and only if $0<\lim _{n} n^{-1} \sum_{i=0}^{n-1} \varepsilon_{i}$. 
I. Let $\mathrm{\textrm {L }}\left(N_{i}\right)=K\left(g^{i}\left(r_{j}\right)\right)$ for each $i \in \mathbb{N}_{0}$, i.e. let all $\varepsilon_{i}$ be zero. Recall that $g_{T}$ is affine on each interval $N_{i}$; if we put

$$
J_{k}=\left\{x \in N_{0}: g_{T}^{i}(x) \in N_{i} \text { for } i \in\{0, \ldots, k\}\right\}, \quad k \in \mathbb{N}_{0},
$$

then $J_{k}$ is a closed subinterval of $N_{0}, J_{k} \supset J_{k+1}$ and $y \in \bigcap J_{k}$. Without loss of generality we can assume that $N_{0}=M_{m}$. Since $(R, g)$ is a twist cycle and $\beta>1$ we have $N_{1}=M_{m+1}$ and $N_{2}<M_{m}$. At the same time $M_{m+1} \rightarrow N_{2} \cup \ldots \cup M_{m}$. This implies that $\left|J_{2}\right|=c\left|N_{0}\right|$ for some $c \in(0,1)$. Since the sequences $\left\{\mathrm{E}\left(N_{i}\right)\right\}_{i \in \mathbb{N}_{0}},\left\{N_{i}\right\}_{i \in \mathbb{N}_{0}}$ are periodic with period $m+n$, for each $l \in \mathbb{N}$ we obtain $\left|J_{2+l(m+n)}\right| \leq c^{l+1}\left|N_{0}\right|$. Thus, the intersection $\bigcap J_{k}$ contains exactly one point $r_{m} \in R$-a contradiction with the choice of the generic point $y \in G(\nu)$ with $\operatorname{supp} \nu \backslash T \neq \emptyset$. This shows that some $\varepsilon_{i}=\varepsilon\left(y_{i}\right)$ has to be positive.

II. Thus we can find a neighborhood $U\left(y_{i}\right)$ of $y_{i}$ satisfying

$$
g_{T}\left(U\left(y_{i}\right)\right) \subset N_{i+1}, \quad \nu\left(\operatorname{bd} U\left(y_{i}\right)\right)=0, \quad \nu\left(U\left(y_{i}\right)\right)=\delta>0 .
$$

It is clear that if for some $k \in \mathbb{N}$ we have $y_{k} \in U\left(y_{i}\right)$ then also $\varepsilon\left(y_{k}\right)=\varepsilon\left(y_{i}\right)$. Since for the $\nu$-generic point $y$ we have $\lim _{n \rightarrow \infty} n^{-1} \#\left\{j \leq n-1: y_{j} \in\right.$ $\left.U\left(y_{i}\right)\right\}=\delta$, we also have $\lim _{n} n^{-1} \sum_{j=0}^{n-1} \varepsilon_{j} \geq \delta \varepsilon\left(y_{i}\right)>0$, hence $\gamma<\beta$.

We have proved that $E(\nu)<\beta$ whenever $\nu \in \mathcal{M}_{\mathrm{e}}\left(g_{T}\right)$ and $\operatorname{supp} \nu \backslash T \neq \emptyset$. This finishes the proof of this part.

4. Strict ergodicity of irrational twist systems. By (1) it is natural to view an irrational twist system as a modification of the irrational circle rotation. Unfortunately, we lack a more detailed knowledge of their relation, for instance how to construct all irrational twist systems. Of course, one can "derive" some properties of irrational twist systems using the known ones for twist cycles [BK2].

It was shown in [BK3] that each irrational twist system is minimal. In this section we will prove even more. Just as any irrational circle rotation, every irrational twist system turns out to be strictly ergodic.

For $\beta>0$ define $R_{\beta}:[0,1) \rightarrow[0,1)$ by

$$
R_{\beta}(x)=x+\frac{1}{1+\beta}(\bmod 1) .
$$

The following lemma states one known property of $R_{\beta}$.

Lemma 4.1 ([DGS]). The Lebesgue measure $\lambda$ is in $\mathcal{M}_{\mathrm{e}}\left(R_{\beta}\right)$ if and only if $\beta$ is irrational.

Let $(T, g)$ be an irrational twist $(\beta)$-system, and again denote by $K$ its normalized $(\beta)$-coding. We define the map $L: T \rightarrow[0,1)$ by $L(x)=$ $K(x)(\bmod 1), x \in T(\mathrm{cf}$. [BM, Proposition 5.9]). If $Z$ denotes the set of 
all endpoints of contiguous intervals of $T$ then the sets $Z$ and $L(Z)$ are countable, and $C \subset[0,1)$ defined by

$$
C=\bigcap_{n=0}^{\infty} R_{\beta}^{n}([0,1) \backslash L(Z))
$$

has Lebesgue measure 1. In Lemmas 4.2-4.3 we show that - in a sense - any irrational twist $(\beta)$-system is an extension of $R_{\beta}$ (for the notion of extension see $[\mathrm{DGS}])$.

Lemma 4.2. Let $(T, g)$ be an irrational twist $(\beta)$-system. Then:

(i) $L \circ g=R_{\beta} \circ L$ on $T$.

(ii) $L(T)=[0,1)$.

(iii) For each $x \in[0,1), L^{-1}(x)$ is finite.

(iv) For $x_{1}, x_{2} \in T$, if $L\left(x_{1}\right)=L\left(x_{2}\right) \in C$ then there exists $n \in \mathbb{N}$ such that $g^{n}\left(x_{1}\right)=g^{n}\left(x_{2}\right)$.

Proof. Properties (i)-(iii) follow from Proposition 2.2.

Let us prove (iv). From (i) and definition (6) of $C$ we obtain

$$
\left(\operatorname{orb}\left(g, x_{1}\right) \cup \operatorname{orb}\left(g, x_{2}\right)\right) \cap Z=\emptyset .
$$

Assume that $L\left(x_{1}\right)=L\left(x_{2}\right)$, i.e. $\left\{K\left(g^{i}\left(x_{2}\right)\right)-K\left(g^{i}\left(x_{1}\right)\right): i \in \mathbb{N}\right\} \subset \mathbb{Z}$. Notice that by (7) and Proposition 2.2(iii) we have, for any $i \in \mathbb{N}$,

$$
c \notin \operatorname{conv}\left\{g^{i}\left(x_{1}\right), g^{i}\left(x_{2}\right)\right\} \& K\left(g^{i}\left(x_{1}\right)\right)=K\left(g^{i}\left(x_{2}\right)\right) \Rightarrow g^{i}\left(x_{1}\right)=g^{i}\left(x_{2}\right) .
$$

Suppose that (iv) does not hold, i.e. $g^{i}\left(x_{1}\right) \neq g^{i}\left(x_{2}\right)$ for each $i \in \mathbb{N}$. Since $\beta>1$ without loss of generality we can assume that $x_{1}, x_{2} \in[\min T, c$ ) (see Remark 1.3) and $x_{1}<x_{2}$; the monotonicity of the coding $K$ gives

$$
K\left(x_{1}\right)<K\left(x_{2}\right) .
$$

In what follows we will say that some couple $\left(g^{i}\left(x_{1}\right), g^{i}\left(x_{2}\right)\right), i \in \mathbb{N}_{0}$, is ordered if $g^{i}\left(x_{1}\right), g^{i}\left(x_{2}\right) \in[\min T, c)$ and $K\left(g^{i}\left(x_{1}\right)\right)<K\left(g^{i}\left(x_{2}\right)\right)$. Thus the couple $\left(x_{1}, x_{2}\right)$ is ordered.

We know from Proposition 2.2(iii) that $(T, g)$ is minimal. Take $\varepsilon \in$ $(0, \beta /(1+\beta))$. The coding $K$ is normalized and we can consider $i \in \mathbb{N}$ for which $K\left(g^{i}\left(x_{2}\right)\right)<\varepsilon$; since (iv) does not hold, from (8) we have

$$
K\left(g^{i}\left(x_{1}\right)\right)>K\left(g^{i}\left(x_{2}\right)\right) \text {. }
$$

But from the definition of $K$ we can see that

$$
\left(K\left(g^{i+1}\left(x_{1}\right)\right)-K\left(g^{i+1}\left(x_{2}\right)\right)\right)-\left(K\left(g^{i}\left(x_{1}\right)\right)-K\left(g^{i}\left(x_{2}\right)\right)\right) \in\{-1,0,1\} .
$$

This gives $K\left(g^{j}\left(x_{1}\right)\right)=K\left(g^{j}\left(x_{2}\right)\right)$ for some $j \in\{1, \ldots, i-1\}$. Let $i_{0} \in \mathbb{N}$ be the least number with this property. By (8), $c \in \operatorname{conv}\left\{K\left(g^{i_{0}}\left(x_{1}\right)\right)\right.$, $\left.K\left(g^{i_{0}}\left(x_{2}\right)\right)\right\}$. We distinguish two possibilities: 
I. If $K\left(g^{i_{0}}\left(x_{1}\right)\right)<c<K\left(g^{i_{0}}\left(x_{2}\right)\right)$ then (8) gives $K\left(g^{i_{0}-1}\left(x_{1}\right)\right)-$ $K\left(g^{i_{0}-1}\left(x_{2}\right)\right)=1>0$, which together with (9) contradicts the choice of $i_{0}$. So this situation is not possible.

II. Thus $K\left(g^{i_{0}}\left(x_{2}\right)\right)<c<K\left(g^{i_{0}}\left(x_{1}\right)\right)$. Since $\beta>1$ there is the least positive integer $j_{0}$ such that $g^{i_{0}+j_{0}}\left(x_{1}\right), g^{i_{0}+j_{0}}\left(x_{2}\right) \in[\min T, c)$; we have excluded the possibility $K\left(g^{i_{0}+j_{0}}\left(x_{1}\right)\right)=K\left(g^{i_{0}+j_{0}}\left(x_{2}\right)\right)$, hence by the definition of $K$ the couple $\left(g^{i_{0}+j_{0}}\left(x_{1}\right), g^{i_{0}+j_{0}}\left(x_{2}\right)\right)$ is ordered. Notice that

$$
\begin{aligned}
& \left\{K\left(g^{i}\left(x_{2}\right)\right)-K\left(g^{i}\left(x_{1}\right)\right): i=0, \ldots, i_{0}-1\right\} \subset \mathbb{N}, \\
& \left\{K\left(g^{i}\left(x_{2}\right)\right)-K\left(g^{i}\left(x_{1}\right)\right): i=i_{0}, \ldots, i_{0}+j_{0}\right\} \subset\{0,1\} .
\end{aligned}
$$

Now repeating the previous procedure for the points $g^{i_{0}+j_{0}}\left(x_{1}\right), g^{i_{0}+j_{0}}\left(x_{2}\right)$ in place of $x_{1}, x_{2}$ we can find the least positive integer $i_{1}>i_{0}+j_{0}$ such that $K\left(g^{i_{1}}\left(x_{1}\right)\right)=K\left(g^{i_{1}}\left(x_{2}\right)\right)$ and the least positive integer $j_{1}$ for which the couple $\left(g^{i_{1}+j_{1}}\left(x_{1}\right), g^{i_{1}+j_{1}}\left(x_{2}\right)\right)$ is ordered; etc.

Summarizing, if (iv) does not hold we obtain

$$
\left\{K\left(g^{i}\left(x_{2}\right)\right)-K\left(g^{i}\left(x_{1}\right)\right): i=0,1, \ldots\right\} \subset \mathbb{N}_{0},
$$

which is impossible by (10). This finishes the proof.

Let $(T, g)$ be an irrational twist $(\beta)$-system. Using Proposition 2.2 we define $m_{T}=[\max K(T)]+1$, where $[x]$ is the integer part of a real number $x$, and $\mathcal{V}=\{0,1\}^{2 m_{T}}$; we define $\eta:[0,1) \rightarrow \mathcal{V}$ by setting $\eta(x)=\left(v_{1}, \ldots, v_{2 m_{T}}\right)$ where for $i \in\left\{1, \ldots, m_{T}\right\}$ (see Remark 1.3),

$$
v_{i}= \begin{cases}1, & {[i-1, i) \cap K\left(T_{\mathrm{L}} \cap L^{-1}(x)\right) \neq \emptyset,} \\ 0, & \text { otherwise }\end{cases}
$$

and similarly for $i \in\left\{m_{T}+1, \ldots, 2 m_{T}\right\}$,

$$
v_{i}= \begin{cases}1, & {\left[i-m_{T}-1, i-m_{T}\right) \cap K\left(T_{\mathrm{R}} \cap L^{-1}(x)\right) \neq \emptyset,} \\ 0, & \text { otherwise. }\end{cases}
$$

We set $C_{k}=\left\{x \in[0,1): \# L^{-1}(x)=k\right\}, k \in \mathbb{N}$. It follows from Lemma 4.2 (ii)-(iii) that $[0,1)=\bigcup_{k \in \mathbb{N}} C_{k}$.

Lemma 4.3. Let $(T, g)$ be an irrational twist $(\beta)$-system. Then $\lambda\left(C_{1}\right)=1$.

Proof. First we show that all sets $C_{k}$ are $\lambda$-measurable.

For each $j \in\left\{0, \ldots, 2 m_{T}\right\}$ define $\mathcal{V}_{j}=\left\{v=\left(v_{1}, \ldots, v_{2 m_{T}}\right) \in \mathcal{V}: j=\right.$ $\left.\sum v_{i}\right\}$. If $\Pi_{i}: \mathcal{V} \rightarrow\{0,1\}$ denotes the usual $i$ th projection then we deduce from Proposition 2.2 that in the relative topology of $[0,1)$,

$$
\forall i \in\left\{1, \ldots, 2 m_{T}\right\}: \quad \eta^{-1} \circ \Pi_{i}^{-1}(a) \text { is } \begin{cases}\text { closed } & \text { if } a=1, \\ \text { open } & \text { for } a=0 .\end{cases}
$$


Now, for $v=\left(v_{1}, \ldots, v_{2 m_{T}}\right) \in \mathcal{V}$,

$$
\eta^{-1}(v)=\bigcap_{i=1}^{2 m_{T}} \eta^{-1} \circ \Pi_{i}^{-1}\left(v_{i}\right)
$$

and $C_{k}=\bigcup_{v \in \mathcal{V}_{k}} \eta^{-1}(v)$, hence all sets $C_{k}$ are $\lambda$-measurable. Since the coding $K$ is monotone and bounded there has to exist $k_{0} \in \mathbb{N}$ such that $C_{k}=\emptyset$ for $k>k_{0}$, i.e. $[0,1)=\bigcup_{k=1}^{k_{0}} C_{k}$. Put $D_{j}=\bigcup_{k \leq j} C_{k}, j \in\left\{1, \ldots, k_{0}\right\}$. Then by Lemma $4.2(\mathrm{i}), R_{\beta}\left(D_{j}\right) \subset D_{j}$ for each $j$ and there exists $j_{0} \in\left\{1, \ldots, k_{0}\right\}$ such that $\lambda\left(C_{j_{0}}\right)=1$.

Let us show that $j_{0}=1$. If $j_{0}>1$ then we would have $\lambda(C \cap \widetilde{C})=1$ for $\widetilde{C}$ defined by

$$
\widetilde{C}=\bigcap_{n=0}^{\infty} R_{\beta}^{n}\left(C_{j_{0}}\right)
$$

and $C$ given by (6); obviously for $x \in C \cap \widetilde{C}$ and two distinct points $x_{1}, x_{2} \in$ $L^{-1}(x)$ we have $g^{i}\left(x_{1}\right) \neq g^{i}\left(x_{2}\right)$ for each $i \in \mathbb{N}$. This contradicts conclusion (iv) of Lemma 4.2. Thus $j_{0}=1$ and the proof of the lemma is finished.

Let $(X, \varrho)$ be a metric space. For $\delta>0$ and $x \in X$ we use the notation $U(x, \delta)=\{y \in X: \varrho(x, y)<\delta\}$. We denote by $B(X)$ the set of all Borel sets in $(X, \varrho)$.

LEMMA 4.4. Let $\mu, \nu$ be two Borel probability measures on a compact metric space $(X, \varrho)$. If there exists $Y \in B(X)$ such that $\mu(Y)=\nu(Y)=1$ and

(11) $\forall x \in Y \exists \delta=\delta(x)>0 \forall M \in B(X): \quad M \subset U(x, \delta) \Rightarrow \mu(M)=\nu(M)$, then $\mu=\nu$.

Proof. Take some $G \in B(X)$ and fix $\varepsilon>0$. Since both measures $\mu, \nu$ are regular we can find some open set $U$ with $X \backslash Y \subset U$ and

$$
\mu(U)+\nu(U)<\varepsilon .
$$

Consider the system $\{U\} \cup\{U(x, \delta(x))\}_{x \in Y}$ of open sets given by (11). Obviously $X=U \cup \bigcup_{x \in Y} U(x, \delta(x))$ and since $X$ is compact we can find $x_{1}, \ldots, x_{n} \in Y$ for which $X=U \cup \bigcup_{i=1}^{n} U\left(x_{i}, \delta\left(x_{i}\right)\right)$. For simplicity put $U_{i}=U\left(x_{i}, \delta\left(x_{i}\right)\right), i \in\{1, \ldots, n\}$, and $U_{n+1}=U$. Define $G_{j}$ by $G_{1}=U_{1} \cap G$ and $G_{j}=U_{j} \cap\left(G \backslash \bigcup_{i=1}^{j-1} U_{i}\right), j \in\{2, \ldots, n+1\}$. Then $G_{j} \in B(X), G_{j} \subset U_{j}$, $G_{j(1)} \cap G_{j(2)}=\emptyset$ for distinct $j(1), j(2)$ and $G=\bigcup_{j=1}^{n+1} G_{j}$. By (11) and (12) we can see that

$$
|\mu(G)-\nu(G)|=\left|\sum_{j=1}^{n+1} \mu\left(G_{j}\right)-\sum_{j=1}^{n+1} \nu\left(G_{j}\right)\right|=\mid \mu\left(G_{n+1}\right)-\nu\left(G_{n+1} \mid<\varepsilon .\right.
$$

Since $\varepsilon$ was arbitrary we obtain $\mu(G)=\nu(G)$ for every $G \in B(X)$. 
We now state the main result of this section. By a portion of $T$ we mean any set $J \cap T$, where $J$ is an open subinterval of $\mathbb{R}$.

TheOREM 4.5. Let $(T, g)$ be an irrational twist $(\beta)$-system. Then $(T, g)$ is strictly ergodic.

Proof. By Proposition 2.2(iii) it is sufficient to show that $\# \mathcal{M}_{\mathrm{e}}(g)=1$.

Let $\mu \in \mathcal{M}_{\mathrm{e}}(g)$. It follows from the minimality of $(T, g)$ guaranteed by Proposition 2.2(iii) that $\mu$ is nonatomic and $\operatorname{supp} \mu=T$.

I. First we show that for any Borel set $N \subset[0,1)$ the equality $\lambda(N)=0$ implies $\mu\left(L^{-1}(N)\right)=0$. Recall that both measures $\lambda$ and $\mu$ are regular [DGS]. Assuming $\lambda(N)=0$, for any positive $\varepsilon$ we can find finitely many intervals $J_{k}, k=1, \ldots, m$, such that $J=\bigcup_{k=1}^{m} J_{k} \supset N$ and $\lambda(J)=\varepsilon$. By Lemmas 4.1 and 4.2 for a $\mu$-generic point $x \in T$ we obtain

$$
\begin{aligned}
\varepsilon & =\lambda(J)=\lim _{n \rightarrow \infty} \frac{\#\left\{i<n: R_{\beta}^{i}(L(x)) \in J\right\}}{n}=\lim _{n \rightarrow \infty} \frac{\#\left\{i<n: L\left(g^{i}(x)\right) \in J\right\}}{n} \\
& =\lim _{n \rightarrow \infty} \frac{\#\left\{i<n: g^{i}(x) \in L^{-1}(J)\right\}}{n}=\mu\left(L^{-1}(J)\right) \geq \mu\left(L^{-1}(N)\right) .
\end{aligned}
$$

This means $\mu\left(L^{-1}(N)\right)=0$.

II. In order to finish the proof we will use Lemma 4.4 putting $X=T$ with the Euclidean metric $\varrho$ and $Y=L^{-1}\left(C_{1} \backslash\{0\}\right)$ (cf. Lemma 4.3).

As above let $\mu \in \mathcal{M}_{\mathrm{e}}(g)$. We know from Lemma 4.3 that $\lambda\left(C_{1} \backslash\{0\}\right)=1$, hence from the first part of this proof we get $\mu(Y)=1$. Fix $x \in Y$. Obviously there are $i_{0} \in\left\{1, \ldots, m_{T}\right\}$ and $S \in\{\mathrm{R}, \mathrm{L}\}$ (see Remark 1.3) such that $x \in T_{S} \cap K^{-1}\left(\left(i_{0}-1, i_{0}\right)\right)$. It follows from the properties of the $(\beta)$-coding $K$ described in Proposition 2.2(iii) and the definition of the map $L$ that for $L(x) \in C_{1}$ we can find an open interval $J \subset[0,1)$ such that $L(x) \in J$ and $L^{-1}(J) \subset T_{S} \cap K^{-1}\left(\left(i_{0}-1, i_{0}\right)\right)$. Then for sufficiently small $\delta=\delta(x)$ and any portion $G \subset(x-\delta(x), x+\delta(x)) \subset L^{-1}(J)$ of $T$ we obtain, from Lemmas 4.1 and 4.2 ,

$$
\begin{aligned}
\lambda(L(G)) & =\lim _{n \rightarrow \infty} \frac{\#\left\{i<n: R_{\beta}^{i}(L(x)) \in L(G)\right\}}{n} \\
& =\lim _{n \rightarrow \infty} \frac{\#\left\{i<n: L\left(g^{i}(x)\right) \in L(G)\right\}}{n} \\
& =\lim _{n \rightarrow \infty} \frac{\#\left\{i<n: g^{i}(x) \in G\right\}}{n}=\mu(G) .
\end{aligned}
$$

Since the measure $\mu$ is regular, (13) implies that $\mu(M)=\lambda(L(M))$ whenever $M \subset(x-\delta(x), x+\delta(x))$ and $M \in B(T)$. Using Lemma 4.4 we can see that $\mu$ is the unique measure in $\mathcal{M}_{\mathrm{e}}(g)$. The proof of the theorem is finished. 


\section{Strictly ergodic twist systems exhibited by continuous maps.} In [BK2], [BK3] and the previous sections we gave a description of basic properties of twist systems. Moreover, Proposition 2.1(ii) states that any continuous interval map $f$ has twist cycles of all rational eccentricities from $(1, E(f))$. However, it is still not clear what can be said about irrational twist systems that are realized by $f$. This gap will be removed by Theorem 5.2 .

Proposition 5.1 ([B3], [BK1]). Let $f \in C(I)$ and assume there is a set $S \subset I$ such that $(S, f)$ is a system. Then for $f_{S} \in C(\operatorname{conv} S)$ and $T \subset \operatorname{conv} S$ such that $\left(T, f_{S}\right)$ is a minimal system there is $T^{\star} \subset \operatorname{conv} S$ for which $\left(T^{\star}, f\right)$ is also a minimal system and

$$
f_{S}^{i}(\min T)<f_{S}^{j}(\min T) \Leftrightarrow f^{i}\left(\min T^{\star}\right)<f^{j}\left(\min T^{\star}\right), \quad i, j \in \mathbb{N}_{0} .
$$

We now state the main result of this part. Note that by Proposition 2.1(ii) for $f \in C(\mathcal{I})$ we have $E(f)>1$ if and only if $f$ has a cycle of odd period greater than one.

Theorem 5.2. Let $f \in C(\mathcal{I})$ and $\beta \in(1, E(f))$. Then $f$ has a strictly ergodic twist $(\beta)$-system.

Proof. Since every cycle is a strictly ergodic system, the conclusion holds for $\beta$ rational by Proposition 2.1(ii).

So suppose that $\beta$ is irrational and fix some $\alpha \in(\beta, E(f))$ rational. By the above, the map $f$ has a twist $(\alpha)$-cycle $(S, f)$.

I. First we will show that $g=f_{S} \in C(\operatorname{conv} S)$ has some twist $(\beta)$-system. Again by Proposition 2.1(ii) we can consider a sequence $\left\{\beta_{n}\right\}_{n \in \mathbb{N}} \subset(\beta, \alpha)$ of rational numbers and a sequence $\left\{T_{n}\right\}_{n \in \mathbb{N}}$ of finite subsets of $I=\operatorname{conv} S$ such that $\lim _{n \rightarrow \infty} \beta_{n}=\beta$ and

$$
\forall n \in \mathbb{N}: \quad\left(T_{n}, g\right) \text { is a twist }\left(\beta_{n}\right) \text {-cycle. }
$$

Recall that $(S, g) \in \mathcal{U}$ and $\operatorname{Fix}(g)=\{c\}$. Using Remark 1.5 without loss of generality we can assume that for each $n$,

$$
E\left(T_{n}, g\right)=\frac{\#\left(T_{n} \cap[\min S, c)\right)}{\#\left(T_{n} \cap(c, \max S]\right)} .
$$

By Lemma 2.3 and the monotonicity of each $\left(\beta_{n}\right)$-coding of $\left(T_{n}, g\right)$ (for each $n$ we have $\left.M\left(T_{n}, g\right) \leq M(S, g)\right)$, we obtain $(S \cup\{c\}) \cap D=\emptyset$ where $D=\bigcap_{k=1}^{\infty} \overline{\bigcup_{n=k}^{\infty} T_{n}}$. Clearly $g(D) \subset D$, i.e. $(D, g)$ is a system and we can consider $T \subset D$ such that $(T, g)$ is a minimal system [DGS].

Now we are going to show that $(T, g)$ is a twist $(\beta)$-system. By $(1)$ and Proposition 2.2(ii) it is sufficient to verify that for $t=\min T$ the $(\beta)$-code $K_{t}$ given by $K_{t}(t)=0$ is monotone. Suppose to the contrary that there are $y=g^{i}(t), z=g^{j}(t) \in \operatorname{orb}(g, t)$ for which $y \in \operatorname{conv}\{z, c\}$ and at the same time $K_{t}(y, \beta)<K_{t}(z, \beta)$ (see Remark 1.6). Then also $K_{t}\left(y, \beta_{n}\right)<$ $K_{t}\left(z, \beta_{n}\right)$ for each $n>n_{0}$ with $n_{0}$ sufficiently large. Obviously there are 
some $n>n_{0}, \tilde{t} \in T_{n}$ close to $t$ and $\widetilde{y}=g^{i}(\widetilde{t}), \widetilde{z}=g^{j}(\widetilde{t}) \in \operatorname{orb}(g, \widetilde{t})$ satisfying $\widetilde{y} \in \operatorname{conv}\{\widetilde{z}, c\}$ and $K_{\widetilde{t}}\left(\widetilde{y}, \beta_{n}\right)<K_{\widetilde{t}}\left(\widetilde{z}, \beta_{n}\right)$ - a contradiction with (15), (16) and Proposition 2.1(i). Thus, the $(\beta)$-code $K_{t}$ of $t \in T$ is monotone and $f_{S}=g$ has a twist $(\beta)$-system $(T, g)$.

II. In order to finish the proof we will use Proposition 5.1. By its conclusion there is a set $T^{\star} \subset I$ such that $\left(T^{\star}, f\right)$ is minimal and for $t$ as above and $t^{\star}=\min T^{\star}$ with the help of (14) we obtain

$$
f_{S}^{i}(t)<f_{S}^{j}(t) \Leftrightarrow f^{i}\left(t^{\star}\right)<f^{j}\left(t^{\star}\right), \quad i, j \in \mathbb{N}_{0} .
$$

Now the reader can verify that the $(\beta)$-code $K_{t^{\star}}$ of $t^{\star}$ is monotone, i.e. the map $f$ has a twist $(\beta)$-system $\left(T^{\star}, f\right)$. This proves the theorem.

\section{References}

[ALM] L. Alsedà, J. Llibre and M. Misiurewicz, Combinatorial Dynamics and Entropy in Dimension One, Adv. Ser. Nonlinear Dynam. 5, 2nd ed., World Sci. Singapore, 2000 .

[BCp] L. S. Block and W. A. Coppel, Dynamics in One Dimension, Lecture Notes in Math. 1513, Springer, Berlin, 1992.

[Bl1] A. Blokh, Rotation number for unimodal maps, MSRI, 1994, Preprint \# 58-94.

[Bl2] - On rotation intervals for interval maps, Nonlinearity 7 (1994), 1395-1417.

[B13] - The spectral decomposition for one-dimensional maps, Dynamics Reported 4 (1995), 1-59.

[Bl4] -, Decomposition of dynamical systems on an interval, Russian Math. Surveys 38 (1983), 133-134.

[Bl5] -, Functional rotation numbers for one-dimensional maps, Trans. Amer. Math. Soc. 347 (1995), 499-514.

[BM] A. Blokh and M. Misiurewicz, Rotating an interval and a circle, ibid. 351 (1999), 63-78.

[B1] J. Bobok, On entropy of patterns given by interval maps, Fund. Math. 162 (1999), $1-36$.

[B2] - On the topological entropy of green interval maps, J. Appl. Anal. 7 (2001), 107-112.

[B3] - Forcing relation on minimal interval patterns, Fund. Math. 169 (2001), 161173.

[BK1] J. Bobok and M. Kuchta, Invariant measures for maps of the interval that do not have points of some period, Ergodic Theory Dynam. Systems 14 (1994), 9-21.

[BK2] - - , X-minimal patterns and a generalization of Sharkovskiu's theorem, Fund. Math. 156 (1998), 33-66.

[BK3] - - , Irrational twist systems for interval maps, Real Anal. Exchange 27 (2002), to appear.

[DGS] M. Denker, C. Grillenberger and K. Sigmund, Ergodic Theory on Compact Spaces, Lecture Notes in Math. 527, Springer, Berlin, 1977.

[MN] M. Misiurewicz and Z. Nitecki, Combinatorial patterns for maps of the interval, Mem. Amer. Math. Soc. 456 (1990).

[Ph] R. R. Phelps, Lectures on Choquet's Theorem, D. Van Nostrand, Princeton, NJ, 1966 . 
[Pr] C. Preston, Iterates of Piecewise Monotone Mappings on an Interval, Lecture Notes in Math. 1347, Springer, 1988.

[Y] X. Ye, D-function of a minimal set and an extension of Sharkovskii's theorem to minimal sets, Ergodic Theory Dynam. Systems 12 (1992), 365-376.

KM FSv. ČVUT

Thákurova 7

16629 Praha 6, Czech Republic

E-mail: erastus@mbox.cesnet.cz

Received 19 April 2001;

in revised form 22 April 2002 